\title{
Optimal Charging Strategy of Electric Vehicles with Consideration of Battery Storage
}

\author{
Dazhong Zou' ${ }^{1}$ Da Meng ${ }^{2}$, Yinping Dai², Shuai $\mathrm{Lu}^{2}$ and Huan Xie ${ }^{2, *}$ \\ ${ }^{1}$ China Southern Power Grid Electric Vehicle Service Co., Ltd, 518116 Xinghe world phase III, Longgang District, Shenzhen, China \\ ${ }^{2}$ Chengdu HuaMod Technologies Co., Ltd, 610200 Xinglonghu Tianfu New Economic Industrial Park, Chengdu, Sichuan, China
}

\begin{abstract}
The high penetration of electric vehicles (EVs) will increase burden of a power grid. However, the expansion of capacity of distribution facilities is not always possible, especially in some old residential community. This paper proposes to use an optimal charging strategy of EVs with additional battery energy storage (BES) to improve the charging capabilities in a residential community. By modeling the EV charging behavior, the required charging capacity is evaluated using Monte Carlo method and the BES size is determined as the difference between the required capacity and the distribution capacity. An optimal charging strategy is then proposed to reduce the charging cost and ensure the safe running of distribution network.
\end{abstract}

\section{Introduction}

In the context of climate change, most countries' government has come to agreement on taking measures to mitigate the process of global warming and passed the Paris Agreement. Governments have carried out distinguished plans based on their own situation, nonetheless, enhancing the penetration ratio of sustainable energy and electrical vehicles (EVs) is commonly recognized as a paramount approach to reduce $\mathrm{CO} 2$ emission. On the market side, EV sales are growing rapidly in recent years, even under the COVID-19 pandemic; In Europe, the EV sales increased 57\% in the first half-year of 2020 , in contrast to $37 \%$ decrease of total car sales. The vehicle electrification trend will consistently give rise to the tremendous energy demand enhancement for EV charging. Meanwhile, ordinary charging behavior causes load peaks that traditionally requires utility to increase the capacity of distribution network transformer in order to satisfy this rare demand. The solution is cost-ineffective; an alternative, named coordinated charging, is more economic and has been widely studied in recent years. On the other side, the integration of BES and distributed renewable energy, especially, photovoltaic (PV) in a EV charging station would further reduce the impact to the utility grid and is regarded as a promising business model with the grid parity of PV (in certain sunlight-abundant areas) and continuous price drop of renewable energy.

Charging station related topics in the variety of fields such as integration of BES, PV or both [1], optimal sizing of integrated BES [2], PV or both [3], application of energy management strategy(EMS) with BES [4], PV or both [5], and collaborative planning considering distribution network [6] etc. have been widely studied in recent years. The control object of aforementioned EMS mainly refers to BES or PV, in contrast to coordinated charging which regulate the EVs. The common approaches to change the charging behavior are guiding the EV charging through dynamic price [7] or time-of-use (TOU) price [8] and controlling the EV charging directly based on the prediction and optimization results. Moststudied objectives of coordinated charging are increasing the renewable energy penetration or utilization rate (Obj.1), optimizing the energy flow especially reducing the peak-valley difference of a power grid (Obj.2), improving the reliability and stability of power grid (Obj.3) and minimizing the cost of either charging service or charging station (with distribution network considered) (Obj.4). In terms of optimal planning of distribution grid and charging station with coordinated charging strategy (CCS) applied, Duan[9] considers distinct coordinated charging engagement rate and takes the minimum overall cost that consists of initial investment, operation and coordinated charging cost as the optimization target; Aside from taking advantage of TOU price to lead the charging shift to load-valley time, CCS would reimburse the charging-adjusted users from the saving of distribution grid construction, hereby accomplishing a win-win effect. In PV-integrated scenario, Liu [10] researched the CCS aiming at all four objectives as mentioned above, specifically diminishing three-phase unbalance for Obj.3 based on load prediction and realtime TOU price; While Xiong and Yu etc. [11] proposed a real-time CCS based on PV generation. In PV and BES integrated scenario, $\mathrm{Xu}$ [12] studied the effect of CCS on Obj.1, Obj.2 and Obj.4 in a community scenario with different EV penetration; Xing and Xu etc. [13] use 'charging deadline' concept in the PV generation based CCS that achieves Obj.2 and lower the electricity bill of charging station; Li and Zouma etc. [14] set the contract capacity of energy usage as a constraint and use time-of-

\footnotetext{
* Corresponding author: huan.xie@huamod.com
} 
use adjustment (CCS) method that involves in charging priorities with vehicle-to-grid(V2G) operations enabled, as a result of near $50 \%$ maximum charging cost saving.

Regards to BES or PV optimal sizing with CCS in a charging station, Liu and Xue etc. [15] proposed a model to optimize the size of both BES and PV in a fast charging station, but no control on BES. Mirhoseini and Ghaffarzade [16], investigates the optimal battery sizing in a scenario that charging station is not only integrated with PV and BES, but also with demand side management (DSM) strategy implemented on the attached building and V2G and V2B (vehicle-to-building) technology applied. Nevertheless, in their research, the operation of battery depends on TOU price, not prediction-optimization result, and V2G so far is considered not cost-efficient. Besides, majority of studies are conducted under unreal data.

In short, distinct strategies have been proposed for large scale EVs to improve the safe operation of a power grid, however, various charging habit, distinct level of participation in orderly charging will all affect the effect of orderly charging strategies. The degree of how these factors affect the ordered charging has not been well studied. In this work, we propose to use Monte Carlo method to study different affecting factors. After determining BES, a strategy of charging with BES is used to obtain schedule.

\section{Problem formulation}

\subsection{EV charging}

\subsubsection{EV charging demands}

EV charge demands can be modelled by its starting charging time, charging stop time, and charging amount. For residential community, users usually start charging after they come back from work and expect to fully charge their vehicles before leaving home. According to the reference [17], the returning time of EV follows $N\left(\mu_{s}, \sigma_{s}\right)$ which can be approximated as EV charging starting time.

$f_{s}(x)=\left\{\begin{array}{l}\frac{1}{\sqrt{2 \pi}} \exp \left(-\frac{\left(x+24-\mu_{s}\right)^{2}}{2 \sigma_{s}^{2}}\right), 0 \leq x<\mu_{s}-12 \\ \frac{1}{\sqrt{2 \pi}} \exp \left(-\frac{\left(x-\mu_{s}\right)^{2}}{2 \sigma_{s}^{2}}\right), \mu_{s}-12 \leq x<24\end{array}\right.$

Where, $x$ is the starting charging time; $\mu_{\mathrm{s}}=17.53$ and $\sigma_{\mathrm{s}}=2.96$. The report also shows that the SOC of vehicles at the charging starting time:

$$
f_{\text {soc }}(x)=\frac{1}{\sigma_{s o c} \sqrt{2 \pi}} \exp \left[-\frac{\left(x-\mu_{s o c}\right)^{2}}{2 \sigma_{S o c}^{2}}\right]
$$

Where, $x$ is the remaining SOC, $\mu_{\mathrm{soc}}=0.3$ and $\sigma_{\mathrm{soc}}=0.1$.

\subsubsection{Load fluctuation}

The safety of distribution network can be evaluated by its load fluctuation where load includes traditional non-EV charging load and EV charging load.

$$
V=\sum_{i=1}^{n}\left(P_{i}+x_{i}-\bar{P}\right)^{2}
$$

$$
\bar{P}=\frac{1}{n} \sum_{i=1}^{n}\left(P_{i}+x_{i}\right)
$$

Where, $P_{i}$ and $x_{i}$ are non-EV charging power and EV charging power in the $i$ th time interval and the number of EVs and BES in the market, respectively. $\bar{P}$ is the average power. $n$ is the number of time intervals. Considering the load fluctuation in a day and one hour as the time interval, $n$ equals 24 .

\subsubsection{EV charging cost}

Considering the time-of-use electricity price, the EV charging cost can be described as follows:

$$
C_{e}=\sum_{i=1}^{n} \sum_{j=1}^{m_{i}} p_{i}^{e} x_{i j}
$$

Where, $x_{i j}$ is the charging power of $j$ th $\mathrm{EV}$ in the $i$ th time interval. $m_{j}$ is the number of EVs in the $i$ th time interval. $p_{i}^{e}$ is the electricity price in the $i$ th time interval. $n$ is the number of time intervals. Considering the load fluctuation in a day and one hour as the time interval, $n$ equals 24 .

\subsection{BES sizing}

To evaluate the effect of EV charging loads on the burden of the distribution network, Monte Carlo method is used to compute the quantity of the EV charging loads. Because of the random factors EV charging start time and charging quantity, a set of possible load curves of EV charging is generated. The total load of the distribution network is computed by summing of EV charging loads and non-EV loads.

Using the EV loads generated by Monte Carlo method, optimal schedule of EV loads aiming to reduce the load variance is applied to evaluate if there is enough charging capacity. If the capacity of the transformer cannot meet the charging demand with the optimal scheduling charging strategy applied, the shortage of charging capacity can be met by BES.

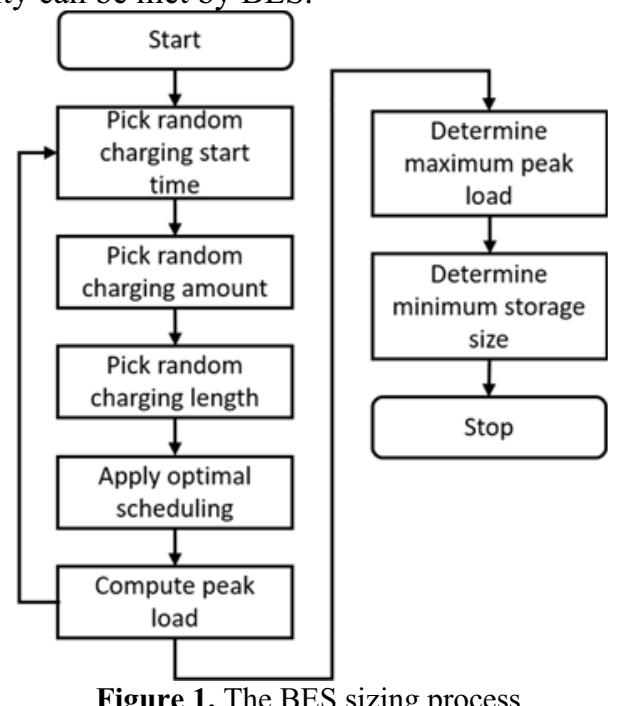

The required amount of BES is computed as the extra EV charging capacity required under orderly charging. The BES sizing process is shown in Figure 1. 


\subsection{Optimal EV charging with BES}

After determining the BES size, the problem becomes how to do optimal scheduling of EC charging and BES charging/discharging to ensure safe running of distribution network and meet the EV charging capacity at the same time. The scheduling strategies are different depending on the actual load level. When both traditional non-EV load and EV charging load are high and the capacity of distribution transformers cannot meet the total load, the schedule of EV charging and BES should be optimized to reduce the load fluctuation. When the total load is much less than the distribution capacity, the goal of optimization is to reduce the charging cost.

Considering the BES, the load fluctuation can be described as:

$$
\begin{gathered}
V_{B E S}=\sum_{i=1}^{n}\left(P_{i}+x_{i}+b_{i}-\bar{P}\right)^{2} \\
\bar{P}_{B E S}=\frac{1}{n} \sum_{i=1}^{n}\left(P_{i}+x_{i}+b_{i}\right)
\end{gathered}
$$

Where $P_{i}, x_{i}$ and $b_{i}$ are non-EV charging power, EV charging power and BES power in the $i$ th time interval, the number of EVs and BES in the market, respectively. $\bar{P}$ is the average power. $n$ is the number of time intervals. Considering the load fluctuation in a day and one hour as the time interval, $n$ equals 24 .

The scheduling model of EV charging and BES power becomes:

$$
\begin{aligned}
& \min \lambda_{\mathrm{e}} C_{e}+\lambda_{v} V \\
& \lambda_{\mathrm{e}}+\lambda_{v}=1 \\
& \text { s.t. } \\
& \sum_{i=1}^{n} \sum_{j=1}^{m_{i}} x_{i j}=C_{E V} \\
& L_{i} \leq x_{i}, i=1, \cdots n \leq U_{i} \\
& L_{B E S} \leq b_{i}, i=1, \cdots n \leq U_{B E S} \\
& 0 \leq S O C_{i}, i=1, \cdots n \leq S O C_{\text {Max }}
\end{aligned}
$$

Where, $C_{E V}$ is the total charge quantity, $L_{i}$ and $U_{i}$ are the minimum and maximum $\mathrm{EV}$ charging power respectively. $L_{i}$ and $U_{i}$ are BES minimum and maximum power. $S O C_{i}$ is the BES SOC at $i$ th time interval. $S O C_{\text {Max }}$ is the maximum SOC of BES.

In practice, the scheduling process starts with determining if there will be transformer overload and the optimization object will be adjusted accordingly. A process of optimal scheduling is shown in Figure 2.

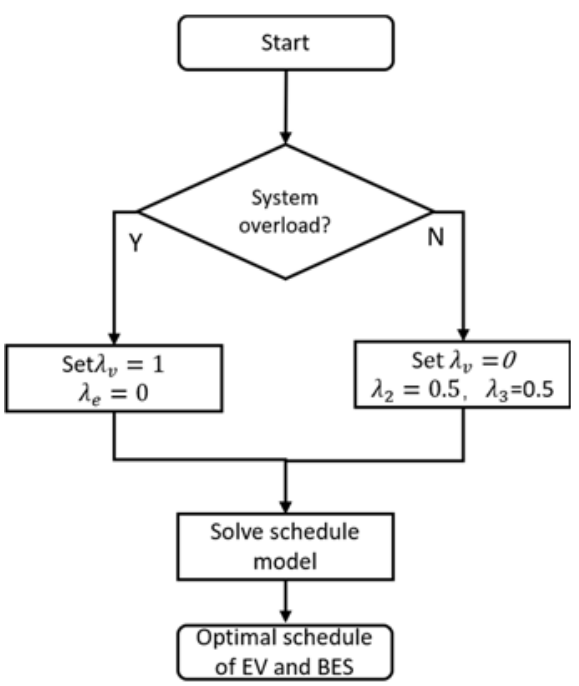

Figure 2. The process of optimal scheduling of EV charging and BES.

\section{Case studies}

\subsection{Study setup}

A residential area with 1000 households is used to demonstrate the BES sizing process and the optimal EV charging process, where the peak non-EV load is about $1000 \mathrm{kVA}$ and the distribution capacity is $1200 \mathrm{kVA}$.

In this paper, the time interval of micro-market is set to 1 hour. Different level of EV penetrations will be studied by assuming $10 \%, 20 \%$ and $50 \%$ of residents own EVs. The TOU electricity price is shown in Table 1.

Table 1. Electricity price.

\begin{tabular}{|c|c|}
\hline Hour & price $(¥ / \mathrm{kWh})$ \\
\hline $1 \sim 8$ & 0.3301 \\
\hline $9 \sim 12,18 \sim 21$ & 1.1507 \\
\hline $13 \sim 17,22 \sim 24$ & 0.6904 \\
\hline
\end{tabular}

\subsection{BES sizing results}

The Monte Carlo simulation results of 30 runs for 200 EVs are shown in Figure 3. It can be seen that the peak EV charging load varies and there is about $100 \mathrm{~kW}$ difference of peak charging load in these runs. The following sections will use the average of these runs to study the effect of EV charging on distribution network, and peak charging variance is about $[-50,50]$. 


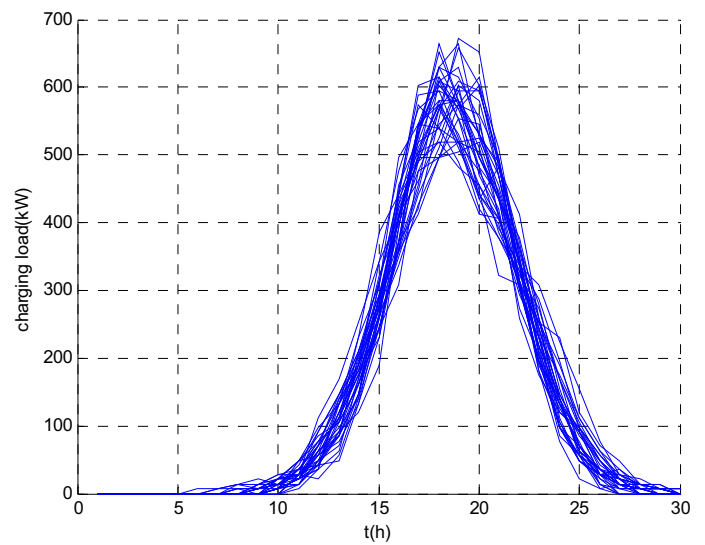

Figure 3. The Monte Carlo simulation results of EV charging demands.

Comparing the EV charging curve to those of non-EV load, it can be seen that EV load and non-EV peak around 8PM. Without the optimal scheduling of EV charging loads, the result in Figure 4 shows that the distribution capacity cannot meet the EV charging demands. About $100 \mathrm{~kW}$ extra charging capacity is needed.

The overload level can be reduced by scheduling EV charging loads, however, it is shown in Figure 5 that when the penetration level is about $50 \%$, i.e., $500 \mathrm{EVs}$, there is still shortage of charging capacities. For the safe operation of distribution network, an extra of $50 \mathrm{~kW}$ charging capacity is needed.

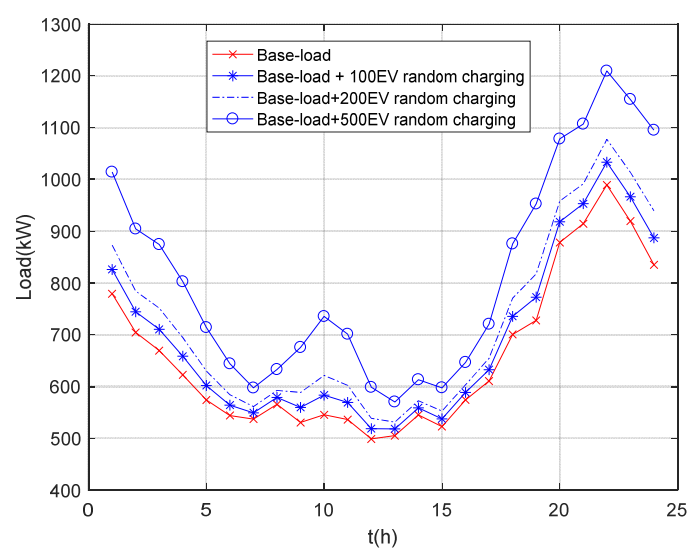

Figure 4. The load level of EV charging demands in random charging.

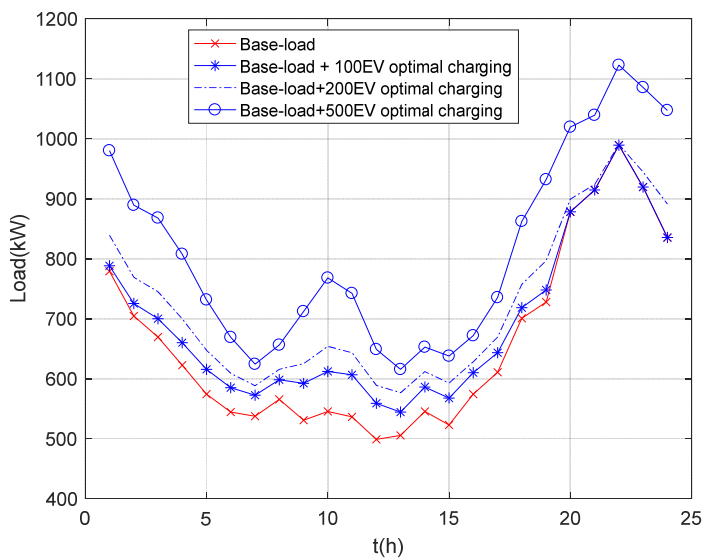

Figure 5. The load level of EV charging demands in random charging.

\subsection{Results of optimal charging with BES}

Results in Figure 6 shows the optimal scheduling of EV charging with $50 \mathrm{~kW}$ BES and $1000 \mathrm{kWh}$. From the results, it can be seen that BES can help to meet all charging requirements even at high EV penetration. This shows BES has more scheduling flexibility comparing to scheduling of EV charging. The charging capacity in a distribution network can be expanded by installing a certain amount of BES.

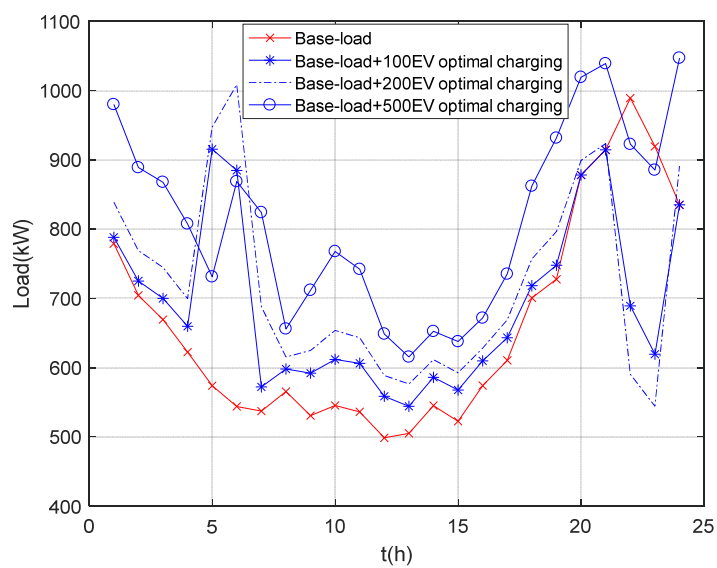

Figure 6. Schedule of EV charging and BES when system capacity is not enough.

It should be pointed out when the load level of a residential area is high, the scheduling of EV charging is to reduce the charging cost. Figure 7 shows a scheduling result for 200 EVs during a day when system has enough capacity. Considering the TOU price, the charging cost reduces from $0.7332 ¥ / \mathrm{kWh}$ to $0.7056 ¥ / \mathrm{kWh}$.

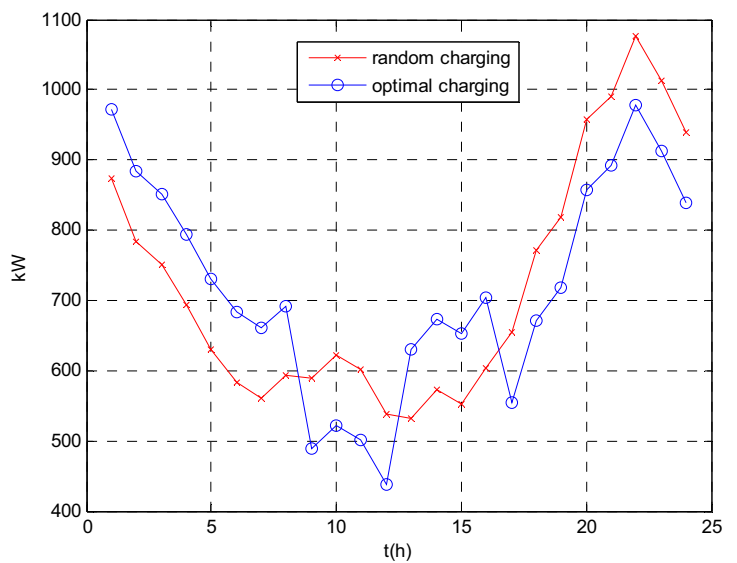

Figure 7. Schedule of EV charging and BES when system capacity is enough.

\section{Conclusion}

To solve the conflict between the increasing charging demands and limited transformer capacity, an optimal scheduling strategy of EV charging and BES is proposed. The whole process starts with evaluating the shortage of charging capacity and determining the BES capacity. The model for optimal scheduling of EV charging with BES is defined and verified using data for a residential area.

A scene of residential area is set up to demonstrate the battery sizing process and optimal scheduling of $\mathrm{EV}$ 
charging with BES. The results show that BES can help to increase the EV charging capacity. This is important especially for old residential area where the expansion of distribution capacity is expensive or impossible.

\section{Acknowledgement}

The project number of this paper is 2020800201 .

\section{References}

1. Y. He, Y. Zhang, Y. Wang, S. Jin, Energy storage Capacity Configuration of PV-integrated EV Charging Station Considering Load Optimization, Modern Electric Power, 36, 76-81 (2019)

2. A. Hussain, V. Bui, J. Baek, H. Kim, Stationary Energy Storage System for Fast EV Charging Stations: Optimality Analysis and Results Validation, Energies, 13, 230 (2020)

3. J. Liu, Q. Dai, Portfolio Optimization of Photovoltaic/Battery Energy Storage/Electric Vehicle Charging Stations with Sustainability Perspective Based on Cumulative Prospect Theory and MOPSO, Sustainability, 12, 985 (2020)

4. W. Xu, H. Cheng, Z. Bai, C. Miao, F. Sun, Optimal Design and Operation of Energy Storage Power Station under Multi-station Fusion Mode, Distribution \& Utilization, 36, 84-91 (2019)

5. J. Yang, L. Wu, Z. Wang, The Operation Control of the PV-Battery-EV Charging Power Plant at Expressway Service Area, Dongfang electric review, 33, 13-18 (2019)

6. Y. Zhang, Z. Chen, R. Duan, X. Huang, Collaborative planning of electric vehicle charging station-distribution network considering active response, Electric Power Engineering Technology, 38, 71-77 (2019)

7. Y. Niu, H. Li, S. Li, Y. Yang, Considering the Realtime Electricity Price Response, Control Strategy of Electric Vehicles after Distributed Energy Access, Shanghai Energy Conservation, 2, 97-101 (2019)

8. Z. Song, Coordinated scheduling strategy of microgrid with electric vehicles and renewable energy, Yanshan Univerisity (2019)

9. R. Duan, Distribution network considering orderly charging, Southeast University (2019)

10. 10. X. Liu, Optimal Scheduling for Electric Vehicles in a Distribution System with Photovoltaic Units and Planning for Charging Facilities, Zhejiang University (2019)

11. W. Xiong, X. Yu, Y. Yang, Energy Management Strategy of Photovoltaic Charging Station for Electric Vehicles in Commercial Area, IOP Conference Series: Materials Science and Engineering, 452 (2018)

12. L. Xu, Study on Coordinated Charging Strategy of Electric Vehicles with Consideration of Renewable
Energy Involvement, North China Electric Power University (2019)

13. H. Xing, Q. Xu, X. Ren, Y. Shen, H. Liu, A Timephased Order Charging Strategy for an Electric Vehicle Photovoltaic Charging Station, Southern Energy Construction, 7, 112-118 (2020)

14. D. Li, A. Zouma, J. Liao,H. Yang, An energy management strategy with renewable energy and energy storage system for a large electric vehicle charging station, eTransportation, 6, 100076 (2020)

15. G. Liu, Y. Xue, M.S. Chinthavali, K. Tomsovic, Optimal Sizing of PV and Energy Storage in an Electric Vehicle Extreme Fast Charging Station, 2020 IEEE Power \& Energy Society Innovative Smart Grid Technologies Conference (ISGT), 1-5 (2020)

16. P. Mirhoseini, N. Ghaffarzadeh, Economic battery sizing and power dispatch in a grid-connected charging, Journal of Energy Storage, 31, 101651 (2020)

17. H. Su, Z. Liang, Orderly charging control based on peak-valley electricity tariffs for household electric vehicles of residential quarter, Electric Power Automation Equipment, 35, 17-22 (2015) 